\title{
FLUID LIMITS FOR THE QUEUE LENGTH OF JOBS IN MULTISERVER OPEN QUEUEING NETWORKS
}

\author{
SAulius MinkevičIUs ${ }^{1}$
}

\begin{abstract}
The object of this research in the queueing theory is a theorem about the Strong-Law-of-Large-Numbers (SLLN) under the conditions of heavy traffic in a multiserver open queueing network. SLLN is known as a fluid limit or fluid approximation. In this work, we prove that the long-term average rate of growth of the queue length process of a multiserver open queueing network under heavy traffic strongly converges to a particular vector of rates. SLLN is proved for the values of an important probabilistic characteristic of the multiserver open queueing network investigated as well as the queue length of jobs.
\end{abstract}

Keywords. Mathematical models of information systems, performance evaluation, queueing theory, multiserver open queueing network, heavy traffic, limit theorem, queue length of jobs.

Mathematics Subject Classification. 60K25, 60G70, 60F17.

\section{INTRODUCTION}

The paper is devoted to the analysis of queueing systems in the context of the network and communications theory. We investigate SLLN about the queue length of customers (jobs) in a multiserver open queueing network under the conditions of heavy traffic. Queueing networks have been extensively used for the analysis of manufacturing and transportation systems, and for computer and communications networks. Therefore, many approximation methods have emerged, and SLLN is among them.

The investigation of delays arising in communications and computer systems is a very complicated problem which has not yet been solved in the general case.

Received December 6, 2011. Accepted January 7, 2014.

1 Institute of Mathematics and Informatics of VU, Akademijos 4, 08663 Vilnius, Lithuania. minkevicius.saulius@gmail.com 
A valuable progress in this area has been achieved for models based on Kleinrock's hypothesis on the independence of transmission times of messages at different nodes (see $[3-5,20,22,24,39]$ ). The most fruitful approach to the calculation of delays in communication systems is based on limit theorems for heavy traffic and light traffic regimes. Some results have been obtained both for systems with finite (see Kelly [21, 24]) and infinite (see [4, 9, 11, 12, 14, 15, 17, 18, 32, 33, 37-39]) waiting rooms. The theoretical base for heavy traffic limit theorems includes the weak convergence results for stochastic processes (see $[1,2,30,35]$ ), as well as the martingale approach to limit theorems (see $[10,23,26]$ ). The limit theorems usually state that, in the heavy traffic regime, properly normalized random variables (or random processes) describing queue lengths or waiting times converge in distribution to a normal random variable (or certain diffusion processes).

The first results for open queueing networks in heavy traffic were obtained by Iglehart and Whitt $[17,18]$. They considered a single station, multiserver and acyclic networks of queues. The limit process for networks in heavy traffic was described as a complicated functional of multidimensional Brownian motion. Harrison [11] considered the heavy traffic approximation to the stationary distribution of the waiting times in single server queues in series. His limit process was also given as a complicated functional of Brownian motion. In reference [12], Harrison again considered the diffusion approximation to tandem queues, described the limit process and found analytical solutions in several special cases. Reiman [31] proved the heavy traffic limit theorems for the queue length process associated with open queueing networks. These theorems state that the limit process is a reflected Brownian motion on the nonnegative orthant with constant directions to each boundary hyperplane. Harrison and Reiman [14] considered the properties of distribution of the multidimensional reflected Brownian motion. Harrison and Williams [15] also analysed Brownian models of open queueing networks with homogeneous customer populations. Reiman [32] studied a multiclass feedback queue in heavy traffic. A network of priority queues with one bottleneck station in heavy traffic was considered by Reiman and Simon [33]. Note that the theory of heavy traffic analysis is rather well developed for systems that satisfy the Kleinrock hypothesis. Without this hypothesis the complexity of the problem dramatically increases.

Limit theorems (diffusion approximations) and SLLN for the queueing system under the conditions of heavy traffic are closely connected (they belong to the same field of research, i.e., investigations on the theory of queueing systems in heavy traffic). Therefore, first we shall try to trace the development of research on the general theory of a queueing system in heavy traffic. There is a vast literature on the diffusion approximation. Readers are referred to $[7,8,25,39]$ for a general survey of the subject. The present work extends the studies by Iglehart and Whitt $[17,18]$ on a single station of multiserver queues, and by Reiman [31], Johnson [19], Chen and Mandelbaum [6] on networks of single server queues. Other closely related papers are by Harrison and Lemoine [13] on networks of infinite server queues, and Whitt [40] for a $G I / G / \infty$ queue. 
The natural setting for functional limit theorems in this paper is the weak convergence of probability measures on the function space $D[0,1]$. Since an excellent treatment of this subject has been recently published by Billingsley [1], we shall only make a few remarks here about our terminology and notation. Stochastic processes characterizing the queueing system give rise to sequences of random functions in $D$, the space of all right-continuous functions on $[0,1]$ having left limits and endowed with Skorohod metric, $d$. In [1], this metric is denoted by $d_{0}$. With $d, D$ is a complete, separable metric space. Let $\mathcal{D}$ be the class of Borel sets of $D$. Then, if $P_{n}$ and $P$ are probability measures on $\mathcal{D}$ which satisfy

$$
\lim _{n \rightarrow \infty} \int_{D} f \mathrm{~d} P_{n}=\int_{D} f \mathrm{~d} P
$$

for every bounded, continuous, real-valued function $f$ on $D$, we say that $P_{n}$ weakly converges to $P$, as $n \rightarrow \infty$, and write $P_{n} \Rightarrow P$. A random function $X$ is a measurable mapping from some probability space $(\Omega, \mathcal{B}, \mathcal{P})$ into $D$ with the distribution $P=\mathcal{P} X^{-1}$ on $(D, \mathcal{D})$. We say that a sequence of random functions $\left\{X_{n}\right\}$ weakly converges to the random function $X$, and write $X_{n} \Rightarrow X$, if the distribution $P_{n}$ of $X_{n}$ converges to the distribution $P$ of $X$. A sequence of random functions $\left\{X_{n}\right\}$ weakly converges to $X$ in probability if $X_{n}$ and $X$ are defined on a common domain and for all $\varepsilon>0, P\left\{\mathrm{~d}\left(X_{n}, X\right) \geq \varepsilon\right\} \rightarrow 0$. When $X$ is a constant function (not random), the convergence in probability is equivalent to a weak convergence. In such cases, we write $\mathrm{d}\left(X_{n}, X\right) \Rightarrow 0$ or $X_{n} \Rightarrow X$. If $X_{n}$ and $Y_{n}$ have a common domain, we also write $\mathrm{d}\left(X_{n}, Y_{n}\right) \Rightarrow 0, P\left\{\mathrm{~d}\left(X_{n}, Y_{n}\right)>\varepsilon\right\} \rightarrow 0$ as for all $\varepsilon>0$. We also use the uniform metric $\rho$ which is defined by $\rho(x, y)=\sup _{0 \leq t \leq 1}|x(t)-y(t)|$ for $x, y \in D$. Also, note that $\mathrm{d}(x, y) \leq \rho(x, y)$ for $x, y \in D$.

Next, we state two extremely useful theorems for obtaining weak convergence results in applications. The first one has come to be known as the "converging together theorem". For it we assume that $X_{n}$ and $Y_{n}$ are defined on a common domain and take values in a separable metric space $(S, m)$. This result can be found in [1], Theorem 4.1.

\section{Theorem 1.1.}

$$
\text { If } X_{n} \Rightarrow X \text { and } \mathrm{d}\left(X_{n}, Y_{n}\right) \Rightarrow 0 \text {, then } Y_{n} \Rightarrow X \text {. }
$$

Now, suppose $h$ is a measurable mapping of $S$ into $S^{\prime}$, a second metric space with Borel sets $\mathcal{B}$. Each probability measure $P$ on $(S, \mathcal{B})$ induces a unique probability measure $P h^{-1}(A)=P\left(h^{-1} A\right)$ on $\left(S^{\prime}, \mathcal{B}^{\prime}\right)$ for $A \in \mathcal{B}^{\prime}$. Let $D_{h}$ be a set of discontinuities of $h$. The next result, known as a continuous mapping theorem, is an analog of the Mann-Wald theorem for Euclidean spaces (see [1], Thm. 5.1). Define $h \circ X=h(X), X \in D$.

Theorem 1.2.

$$
\text { If } X_{n} \Rightarrow X \text { and } P\left\{X \in D_{h}\right\}=0 \text {, then } h \circ X_{n} \Rightarrow h \circ X \text {. }
$$


In practice we use this result as follows. First we show $X_{n} \Rightarrow X$, often by just quoting the known results. Then, we find an appropriate mapping $h$ which gives us the random elements we are really interested in, $h \circ X_{n}$, and finally, we apply (1.2).

So, we prove here SLLN for the queue length of jobs in a multiserver open queueing network under heavy traffic conditions. The main tool for the analysis of these queueing systems in heavy traffic is a functional limit theorem for a complex renewal process (the proof can be found in [1]).

\section{THE NETWORK MODEL}

Consider a network of $j$ stations, indexed by $j=1,2, \ldots, J$, and the station $j$ with $c_{j}$ servers, indexed by $(j, 1), \ldots,\left(j, c_{j}\right)$. Description of the primitive data and construction of processes of interest are the focus of this section. No probability space will be mentioned in this section, and of course, one can always think that all the variables and processes are defined on the same probability space.

First, $\left\{u_{j}(e), e \geq 1\right\}, j=1,2, \ldots, J$, are $J$ sequences of exogenous interarrival times, where $u_{j}(e) \geq 0$ is the interarrival time between the $(e-1)$-job and the $e$ th job that arrive at the station $j$ exogenously (from the outside of the network). Define $U_{j}(0)=0, U_{j}(n)=\sum_{e=1}^{n} u_{j}(e), n \geq 1$ and $A_{j}(t)=\sup \left\{n \geq 0: U_{j}(n) \leq t\right\}$, where $A_{j}=\left\{A_{j}(t), t \geq 0\right\}$ is called an exogenous arrival process at the station $j$, i.e., $A_{j}(t)$ counts the number of jobs that arrived at the station $j$ from the outside of the network.

Second, $\left\{v_{j k_{j}}(e), e \geq 1\right\}, j=1,2, \ldots, J, k_{j}=1,2, \ldots, c_{j}$, are $c_{1}+\ldots+c_{J}$ sequences of service times, where $v_{j k_{j}}(e) \geq 0$ is the service time for the $e$-th job served by the server $k_{j}$ at the station $j$. Define $V_{j k_{j}}(0)=0, V_{j k_{j}}(n)=\sum_{e=1}^{n} v_{j k_{j}}(e), n \geq 1$ and $x_{j k_{j}}(t)=\sup \left\{n \geq 0: V_{j k_{j}}(n) \leq t\right\}$, where $x_{j k_{j}}=\left\{x_{j k_{j}}(t), t \geq 0\right\}$ is called a service process of the server $k_{j}$ at the station $j$, i.e., $x_{j k_{j}}(t)$ counts the number of services completed by the server $k_{j}$ at the station $j$ during the server's busy time. We write $\mu_{j k_{j}}=\left(E\left[v_{j k_{j}}(e)\right]\right)^{-1}>0, \sigma_{j k_{j}}=D\left(v_{j k_{j}}(e)\right)>0$ and $\lambda_{j}=\left(E\left[u_{j}(e)\right]\right)^{-1}>0, a_{j}=D\left(u_{j}(e)\right)>0, j=1,2, \ldots, k$; with all of these terms assumed finite.

In addition, let $\tilde{\tau}_{j}(t)$ be the total number of jobs routed to the $j$ th station of the network in the interval $[0, t], \tau_{j}(t)$ be the total number of jobs after service departure from the $j$ th station of the network in the interval $[0, t], \tilde{\tau}_{j k_{j}}(t)$ be the total number of jobs routed to the $k_{j}$ server at the $j$ th station of the network in the interval $[0, t]$, let $\tau_{j k_{j}}(t)$ be the total number of customers after service departure from the $k_{j}$ server at the $j$ th station of the network in the interval $[0, t]$, and $\tau_{i j k_{i}}(t)$ be the total number of jobs after service departure from the $k_{i}$ server at the $i$ th station of the network and routed to the $k_{j}$ server of the $j$ th station of the network in the interval $[0, t]$. Let $p_{i j}$ be a probability of the job after service at the $i$ th station of the network routed to the $j$ th station of the network. Denote $p_{i j k_{i}}^{t}=\frac{\tau_{i j k_{i}}(t)}{\tau_{i k_{i}}(t)}$ as part of the total number of jobs which, after service at the $k_{i}$ 
server of the $i$ th station of the network, are routed to the $j$ th station of the network in the interval $[0, t], i, j=1,2, \ldots, J, k_{i}=1, \ldots, c_{i}$ and $t>0$. Also, assume that arrival and service times are independent identically distributed random variables.

The processes of primary interest are the queue length process $Q=\left(Q_{j}\right)$ with $Q_{j}=\left\{Q_{j}(t), t \geq 0\right\}$, where $Q_{j}(t)$ indicates the number of jobs at the station $j$ at time $t$. Now we introduce the following processes $Q_{j k_{j}}=\left\{Q_{j k_{j}}(t), t \geq 0\right\}$, where $Q_{j k_{j}}(t)$ indicates the number of jobs waiting to be served by the server $k_{j}$ of the station $j$ at time $t$; clearly, we have $Q_{j}(t)=\sum_{k_{i}=1}^{c_{j}} Q_{j k_{i}}(t), j=1,2, \ldots, J$.

The dynamics of the queueing system (to be specified) depends on the service discipline at each service station. To be more precise, the "first come, first served" (FCFS) service discipline is assumed for all $J$ stations. When a job arrives at a station and finds more than one server available, it will join one of the servers with the smallest index. We assume that the service station is work conserving; namely, not all servers at a station can be idle when there are customers waiting for service at that station. In particular, we assume that a station must works at its full capacity when the number of jobs waiting is equal to or exceeds the number of servers at that station. Suppose that the queue of jobs in each station of the open queueing network is unlimited.

Let us denote

$$
\begin{aligned}
\beta_{j}= & \sum_{i=1}^{J} \sum_{k_{i}=1}^{c_{i}} \mu_{i k_{i}} \cdot p_{i j}+\lambda_{j}-\sum_{k_{j}=1}^{c_{j}} \mu_{j k_{j}}, \hat{\sigma}_{j}^{2}=\sum_{i=1}^{J} \sum_{k_{i}=1}^{c_{i}} \mu_{i k_{i}}^{3} \cdot \sigma_{i k_{i}} \cdot p_{i j}^{2} \\
& +\lambda_{j}^{3} \cdot a_{j}+\sum_{k_{j}=1}^{c_{j}} \mu_{j k_{j}}^{3} \cdot \sigma_{j k_{j}}>0, j=1,2, \ldots, J
\end{aligned}
$$

Also, we define

$$
\begin{aligned}
\mu_{j k_{j}} & =\left(E v_{j k_{j}}(e)\right)^{-1}>0, \sigma_{j k_{j}}=D v_{j k_{j}}(e)>0, \lambda_{j}=\left(E u_{j}(e)\right)^{-1}>0, \\
a_{j} & =D u_{j}(e)>0, j=1,2, \ldots, J, k_{j}=1,2, \ldots, c_{j} .
\end{aligned}
$$

In this work, we also admit that the following "overload conditions" are fulfilled

$$
\sum_{i=1}^{J} \sum_{k_{i}=1}^{c_{i}} \mu_{i k_{i}, n} \cdot p_{i j}+\lambda_{j}>\sum_{k_{i}=1}^{c_{j}} \mu_{i k_{i}, n}, j=1,2, \ldots, J .
$$

Note that conditions (2.1) quarantee that, with probability one, there exists a queue length of jobs which is constantly growing.

In addition, we assume throughout that

$$
\begin{gathered}
\max _{1 \leq j \leq J} \max _{1 \leq k_{j} \leq c_{j}} \sup _{e \geq 1} E\left(v_{j k_{j}}(e)\right)^{2+\gamma}<\infty \text { for some } \gamma>0, \\
\max _{1 \leq j \leq J} \max _{1 \leq k_{j} \leq c_{j}} \sup _{e \geq 1} E\left(u_{j}(e)\right)^{2+\gamma}<\infty \text { for some } \gamma>0 .
\end{gathered}
$$

Conditions (2.2) and (2.3) imply the Lindeberg conditions for the respective sequences, and are easier to verify in practice (usually $\gamma=1$ work). 


\section{MAin RESUlts}

At first we prove the key lemma.

Lemma 3.1. If $Q_{j}(0)=0, j=1,2, \ldots, J$, then

$$
\left|Q_{j}(t)-\hat{x}_{j}(t)\right| \leq w(t)+\gamma(t),
$$

where

$$
\begin{aligned}
\hat{x}_{j}(t) & =\sum_{i=1}^{J} \sum_{k_{i}=1}^{c_{i}} x_{i k_{i}}(t) \cdot p_{i j}+A_{j}(t)-\sum_{k_{i}=1}^{c_{j}} x_{i k_{i}}(t), w(t) \\
& =\sum_{j=1}^{J} \sum_{i=1}^{J} \sum_{k_{i}=1}^{c_{i}} x_{i k_{i}}(t) . \\
\left|p_{i j k_{i}}^{t}-p_{i j}\right|, \gamma(t) & =\sum_{i=1}^{J} \sum_{k_{i}=1}^{c_{i}} \sup _{0 \leq s \leq t}\left(x_{i k_{i}}(s)-\tau_{i k_{i}}(s)\right)
\end{aligned}
$$

Proof. By definition of the queue of jobs at the stations of the network, we get that, for $j=1,2, \ldots, J, k_{j}=1,2, \ldots, c_{j}$

$$
\begin{aligned}
& Q_{j}(t)=\tilde{\tau}_{j}(t)-\tau_{j}(t)=\sum_{k_{i}=1}^{c_{j}} Q_{i k_{i}}(t)=\sum_{k_{i}=1}^{c_{j}} \tilde{\tau}_{i k_{i}}(t)-\sum_{k_{i}=1}^{c_{j}} \tau_{i k_{i}}(t) \\
& =\sum_{k_{i}=1}^{c_{j}} \tilde{\tau}_{i k_{i}}(t)-\sum_{k_{i}=1}^{c_{j}} x_{i k_{i}}(t)+\sum_{k_{i}=1}^{c_{j}} x_{i k_{i}}(t)-\sum_{k_{i}=1}^{c_{j}} \tau_{i k_{i}}(t) \\
& \leq \sum_{k_{i}=1}^{c_{j}} \tilde{\tau}_{i k_{i}}(t)-\sum_{k_{i}=1}^{c_{j}} x_{i k_{i}}(t)+\sum_{k_{i}=1}^{c_{j}} \sup _{0 \leq s \leq t}\left(x_{i k_{i}}(s)-\tau_{i k_{i}}(s)\right) \\
& =\sum_{i=1}^{J} \sum_{k_{i}=1}^{c_{i}} \tau_{i j k_{i}}(t)+A_{j}(t)-\sum_{k_{i}=1}^{c_{j}} x_{i k_{i}}(t)+\sum_{k_{i}=1}^{c_{j}} \sup _{0 \leq s \leq t}\left(x_{i k_{i}}(s)-\tau_{i k_{i}}(s)\right) \\
& \leq \sum_{i=1}^{J} \sum_{k_{i}=1}^{c_{i}} \tau_{i k_{i}}(t) \cdot \frac{\tau_{i j k_{i}}(t)}{\tau_{i k_{i}}(t)}+A_{j}(t)-\sum_{k_{j}=1}^{c_{j}} x_{j k_{j}}(t)+\sum_{k_{i}=1}^{c_{j}} \sup _{0 \leq s \leq t}\left(x_{i k_{i}}(s)-\tau_{i k_{i}}(s)\right) \\
& \leq \sum_{i=1}^{J} \sum_{k_{i}=1}^{c_{i}} x_{i k_{i}}(t) \cdot p_{i j k_{i}}^{t}+A_{j}(t)-\sum_{k_{j}=1}^{c_{j}} x_{j k_{j}}(t)+\sup _{0 \leq s \leq t}\left(x_{j k_{j}}(s)-\tau_{j k_{j}}(s)\right) \\
& =\sum_{i=1}^{J} \sum_{k_{i}=1}^{c_{i}} x_{i k_{i}}(t) \cdot\left(p_{i j k_{i}}^{t}-p_{i j}+p_{i j}\right)+A_{j}(t)-\sum_{k_{i}=1}^{c_{j}} x_{i k_{i}}(t) \\
& \leq \sum_{i=1}^{J} \sum_{k_{i}=1}^{c_{i}} x_{i k_{i}}(t) \cdot p_{i j}+A_{j}(t)-\sum_{k_{i}=1}^{c_{j}} x_{i k_{i}}(t)+\sum_{i=1}^{J} \sum_{k_{i}=1}^{c_{i}} x_{i k_{i}}(t) \cdot\left|p_{i j k_{i}}^{t}-p_{i j}\right| \\
& +\sum_{k_{i}=1}^{c_{j}} \sup _{0 \leq s \leq t}\left(x_{i k_{i}}(s)-\tau_{i k_{i}}(s)\right)=\hat{x}_{j}(t)+w(t)+\gamma(t), \\
& j=1,2, \ldots, J \text { and } t>0 \text {. }
\end{aligned}
$$


Hence it follows that

$$
Q_{j}(t) \leq \hat{x}_{j}(t)+w(t)+\gamma(t), j=1,2, \ldots, J \text { and } t>0 .
$$

Besides, note that

$$
\begin{aligned}
& Q_{j}(t) \geq \tilde{\tau}_{j}(t)-\sum_{k_{i}=1}^{c_{j}} x_{i k_{i}}(t)=\sum_{i=1}^{J} \sum_{k_{i}=1}^{c_{i}} \tau_{i k_{i}}(t) \cdot p_{i j k_{i}}^{t}+A_{j}(t)-\sum_{k_{i}=1}^{c_{j}} x_{i k_{i}}(t) \\
& =\sum_{i=1}^{J} \sum_{k_{i}=1}^{c_{i}}\left(x_{i k_{i}}(t)+\tau_{i k_{i}}(t)-x_{i k_{i}}(t)\right) \cdot p_{i j k_{i}}^{t}+A_{j}(t)-\sum_{k_{i}=1}^{c_{j}} x_{i k_{i}}(t) \\
& =\sum_{i=1}^{J} \sum_{k_{i}=1}^{c_{i}} x_{i k_{i}}(t) \cdot p_{i j k_{i}}^{t}+\sum_{i=1}^{J} \sum_{k_{i}=1}^{c_{i}}\left(\tau_{i k_{i}}(t)-x_{i k_{i}}(t)\right) \cdot p_{i j k_{i}}^{t}+A_{j}(t) \\
& -\sum_{k_{i}=1}^{c_{j}} x_{i k_{i}}(t)=\sum_{i=1}^{J} \sum_{k_{i}=1}^{c_{i}} x_{i k_{i}}(t) \cdot p_{i j k_{i}}^{t}-\sum_{i=1}^{J} \sum_{k_{i}=1}^{c_{i}}\left(x_{i k_{i}}(t)-\tau_{i k_{i}}(t)\right) \cdot p_{i j k_{i}}^{t} \\
& +A_{j}(t)-\sum_{k_{j}=1}^{c_{j}} x_{j k_{j}}(t) \geq \sum_{i=1}^{J} \sum_{k_{i}=1}^{c_{i}} x_{i k_{i}}(t) \cdot p_{i j k_{i}}^{t}+A_{j}(t)-\sum_{k_{j}=1}^{c_{j}} x_{j k_{j}}(t) \\
& -\sum_{i=1}^{J} \sum_{k_{i}=1}^{c_{i}}\left(x_{i k_{i}}(t)-\tau_{i k_{i}}(t)\right) \geq \sum_{i=1}^{J} \sum_{k_{i}=1}^{c_{i}} x_{i k_{i}}(t) \cdot p_{i j k_{i}}^{t}+A_{j}(t)-\sum_{k_{j}=1}^{c_{j}} x_{j k_{j}}(t) \\
& -\sup _{0 \leq s \leq t} \sum_{i=1}^{J} \sum_{k_{i}=1}^{c_{i}}\left(x_{i k_{i}}(s)-\tau_{i k_{i}}(s)\right) \geq \sum_{i=1}^{J} \sum_{k_{i}=1}^{c_{i}} x_{i k_{i}}(t) \cdot p_{i j k_{i}}^{t}+A_{j}(t) \\
& -\sum_{k_{j}=1}^{c_{j}} x_{j k_{j}}(t)-\sum_{i=1}^{J} \sum_{k_{i}=1}^{c_{i}} \sup _{0 \leq s \leq t}\left(x_{i k_{i}}(s)-\tau_{i k_{i}}(s)\right) \\
& =\sum_{i=1}^{J} \sum_{k_{i}=1}^{c_{i}} x_{i k_{i}}(t) \cdot\left(p_{i j k_{i}}^{t}-p_{i j}+p_{i j}\right)+A_{j}(t)-\sum_{k_{j}=1}^{c_{j}} x_{j k_{j}}(t) \\
& -\sum_{i=1}^{J} \sum_{k_{i}=1}^{c_{i}} \sup _{0 \leq s \leq t}\left(x_{i k_{i}}(s)-\tau_{i k_{i}}(s)\right)=\sum_{i=1}^{J} \sum_{k_{i}=1}^{c_{i}} x_{i k_{i}}(t) \cdot p_{i j}+A_{j}(t) \\
& -\sum_{k_{i}=1}^{c_{j}} x_{i k_{i}}(t)+\sum_{i=1}^{J} \sum_{k_{i}=1}^{c_{i}} x_{i k_{i}}(t) \cdot\left(p_{i j k_{i}}^{t}-p_{i j}\right) \\
& -\sum_{i=1}^{J} \sum_{k_{i}=1}^{c_{i}} \sup _{0 \leq s \leq t}\left(x_{i k_{i}}(s)-\tau_{i k_{i}}(s)\right) \geq \hat{x}_{j}(t) \\
& -\sum_{i=1}^{J} \sum_{k_{i}=1}^{c_{i}} x_{i k_{i}}(t) \cdot\left|p_{i j k_{i}}^{t}-p_{i j}\right|-\sum_{i=1}^{J} \sum_{k_{i}=1}^{c_{i}} \sup _{0 \leq s \leq t}\left(x_{i k_{i}}(s)-\tau_{i k_{i}}(s)\right) \\
& =\hat{x}_{j}(t)-w(t)-\gamma(t), j=1,2, \ldots, J \text { and } t>0 \text {. }
\end{aligned}
$$


Hence it follows that

$$
Q_{j}(t) \geq \hat{x}_{j}(t)-w(t)-\gamma(t)
$$

$j=1,2, \ldots, J$ and $t>0$.

By combining (3.1) and (3.3), we can write

$$
\left|Q_{j}(t)-\hat{x}_{j}(t)\right| \leq w(t)+\gamma(t)
$$

$j=1,2, \ldots, J$ and $t>0$. The proof of the lemma is complete.

Now we use the formulation of Lemmas 3.2 and 3.3 the proof of which is presented in [28].

Lemma 3.2. If conditions (1) are fulfilled, then for every $\varepsilon>0$

$$
P\left(\varlimsup_{t \rightarrow \infty} \frac{\sup _{0 \leq s \leq t}\left(x_{j k_{j}}(s)-\tau_{j k_{j}}(s)\right)}{t}>\varepsilon\right)=0,
$$

$j=1,2, \ldots, J, k_{j}=1,2, \ldots, c_{j}$.

Lemma 3.3. If conditions (1) are fulfilled, then

$$
p_{i j k_{i}}^{t} \Rightarrow p_{i j}, i, j=1,2, \ldots, J, k_{i}=1,2, \ldots, c_{i} .
$$

Finally, applying the results of Lemmas 3.1-3.3, we prove the following theorem about SLLN for the queue length of jobs in multiserver open queueing networks.

Theorem 3.4. If conditions (2.1)-(2.3) are fulfilled, then

$$
\left(\frac{Q_{1}(t)}{t} ; \frac{Q_{2}(t)}{t} \ldots ; \frac{Q_{J}(t)}{t}\right) \Rightarrow\left(\beta_{1} ; \beta_{2} ; \ldots ; \beta_{J}\right) .
$$

Proof. At first we can find that for $\varepsilon>0$

$$
\begin{aligned}
P\left(\left|\frac{Q_{j}(t)}{t}-\beta_{j}\right|>\varepsilon\right) \leq & P\left(\sup _{0 \leq s \leq t}\left|\frac{Q_{j}(s)}{s}-\frac{\hat{x}_{j}(s)}{s}\right|>\frac{\varepsilon}{2}\right) \\
& +P\left(\sup _{0 \leq s \leq t}\left|\frac{\hat{x}_{j}(s)}{s}-\beta_{j}\right|>\frac{\varepsilon}{2}\right), j=1,2, \ldots, J \text { and } t>0 .
\end{aligned}
$$


Denote $c=2 \cdot J^{2} \cdot\left(c_{1}+c_{2}+\cdots+c_{J}\right), W j(t)=Q_{j}(t)-\hat{x}_{j}(t), \quad j=1,2, \ldots, J$ and $t>0$. Let us estimate the first term in inequality (3.6):

$$
\begin{aligned}
& P\left(\frac{\left|W_{j}(t)\right|}{t}>\varepsilon\right) \leq P\left(\frac{\sup _{0 \leq s \leq t}\left\{\sum_{j=1}^{J} \sum_{i=1}^{J} \sum_{k_{i}=1}^{c_{i}} x_{i k_{i}}(s) \cdot\left|p_{i j k_{i}}^{s}-p_{i j}\right|\right\}}{t}>\frac{\varepsilon}{2}\right) \\
& +P\left(\frac{\sup _{0 \leq s \leq t}\left\{\sum_{i=1}^{J} \sum_{k_{i}=1}^{c_{i}} \sup _{0 \leq l \leq s}\left(x_{i k_{i}}(s)-\tau_{i k_{i}}(s)\right\}\right.}{t}>\frac{\varepsilon}{2}\right) \\
& \leq P\left(\frac{\sum_{j=1}^{J} \sum_{i=1}^{J} \sum_{k_{i}=1}^{c_{i}} \sup _{0 \leq s \leq t}\left\{x_{i k_{i}}(s) \cdot\left|p_{i j k_{i}}^{s}-p_{i j}\right|\right\}}{t}>\frac{\varepsilon}{2}\right) \\
& +P\left(\frac{\sum_{i=1}^{J} \sum_{k_{i}=1}^{c_{i}} \sup _{0 \leq s \leq t} \sup _{0 \leq l \leq s}\left\{x_{i k_{i}}(l)-\tau_{i k_{i}}(l)\right\}}{t}>\frac{\varepsilon}{2}\right) \\
& \leq P\left(\frac{\sum_{j=1}^{J} \sum_{i=1}^{J} \sum_{k_{i}=1}^{c_{i}} \sup _{0 \leq s \leq t}\left\{x_{i k_{i}}(s) \cdot\left|p_{i j k_{i}}^{s}-p_{i j}\right|\right\}}{t}>\frac{\varepsilon}{2}\right) \\
& +P\left(\frac{\sum_{i=1}^{J} \sum_{k_{i}=1}^{c_{i}} \sup _{0 \leq s \leq t}\left(x_{i k_{i}}(s)-\tau_{i k_{i}}(s)\right)}{t}>\frac{\varepsilon}{2}\right) \\
& \leq \sum_{j=1}^{J} \sum_{i=1}^{J} \sum_{k_{i}=1}^{c_{i}} P\left(\frac{\sup _{0 \leq s \leq t}\left\{x_{i k_{i}}(s) \cdot\left|p_{i j k_{i}}^{s}-p_{i j}\right|\right\}}{t}>\frac{\varepsilon}{c}\right) \\
& +\sum_{i=1}^{J} \sum_{k_{i}=1}^{c_{i}} P\left(\frac{\sup _{0 \leq s \leq t}\left(x_{i k_{i}}(s)-\tau_{i k_{i}}(s)\right)}{t}>\frac{\varepsilon}{c}\right) \text {, } \\
& j=1,2, \ldots, J, \quad t>0 \text {. }
\end{aligned}
$$


Consequently, it follows from (3.6) that for $\varepsilon>0$

$$
\begin{aligned}
P\left(\frac{\left|W_{j}(t)\right|}{t}>\varepsilon\right) \leq & \sum_{j=1}^{J} \sum_{i=1}^{J} \sum_{k_{i}=1}^{c_{i}} P\left(\frac{\sup _{0 \leq s \leq t}\left\{x_{i k_{i}}(s) \cdot\left|p_{i j k_{i}}^{s}-p_{i j}\right|\right\}}{t}>\frac{\varepsilon}{c}\right) \\
& +\sum_{i=1}^{J} \sum_{k_{i}=1}^{c_{i}} P\left(\frac{\sup _{0 \leq s \leq t}\left(x_{i k_{i}}(s)-\tau_{i k_{i}}(s)\right)}{t}>\frac{\varepsilon}{c}\right), \\
j= & 1,2, \ldots, J, t>0 .
\end{aligned}
$$

Suppose $\mu=\max _{1 \leq j \leq J} \sup _{1 \leq k_{j} \leq c_{j}} \mu_{i k_{i}}<\infty$. Let us estimate the first term in (3.7). We have for $\varepsilon>0$ that

$$
\begin{aligned}
& P\left(\frac{\sup _{0 \leq s \leq t}\left\{x_{i k_{i}}(s) \cdot\left|p_{i j k_{i}}^{s}-p_{i j}\right|\right\}}{t}>\varepsilon\right) \leq P\left(\frac{x_{i k_{i}}(t) \cdot \sup _{0 \leq s \leq t}\left|p_{i j k_{i}}^{s}-p_{i j}\right|}{t}>\varepsilon\right) \\
& \quad \leq P\left(\left\{\frac{x_{i k_{i}}(t) \cdot \sup _{0 \leq s \leq t}\left|p_{i j k_{i}}^{s}-p_{i j}\right|}{t}>\varepsilon\right\} \cap\left\{x_{i k_{i}}(t) \leq \varepsilon \cdot t+\mu_{i k_{i}} \cdot t\right\}\right) \\
& +P\left(\frac{x_{i k_{i}}(t)}{t}-\mu_{i k_{i}}>\varepsilon\right) \leq P\left(\left(\varepsilon \cdot t+\mu_{i k_{i}} \cdot t\right) \cdot \sup _{0 \leq s \leq t}\left|p_{i j k_{i}}^{s}-p_{i j}\right|>\varepsilon \cdot t\right) \\
& \quad+P\left(\frac{x_{i k_{i}}(t)}{t}-\mu_{i k_{i}}>\varepsilon\right) \leq P\left(\sup _{0 \leq s \leq t}\left|p_{i j k_{i}}^{s}-p_{i j}\right|>\frac{\varepsilon}{\varepsilon+\mu_{i k_{i}}}\right) \\
& +P\left(\sup _{0 \leq s \leq t}\left|\frac{x_{i k_{i}}(s)}{s}-\mu_{i k_{i}}\right|>\varepsilon\right) \leq P\left(\sup _{0 \leq s \leq t}\left|p_{i j k_{i}}^{s}-p_{i j}\right|>\frac{\varepsilon}{2 \cdot \mu_{i k_{i}}}\right) \\
& \quad+P\left(\sup _{0 \leq s \leq t}\left|\frac{x_{i k_{i}}(s)}{s}-\mu\right|>\varepsilon\right), i=1,2, \ldots, J, \quad k_{i}=1,2, \ldots, c_{i} .
\end{aligned}
$$

Thus, we achieve for $\varepsilon>0$ that

$$
\begin{aligned}
P\left(\frac{\sup _{0 \leq s \leq t}\left\{x_{i k_{i}}(s) \cdot\left|p_{i j k_{i}}^{s}-p_{i j}\right|\right\}}{t}>\varepsilon\right) \leq & P\left(\sup _{0 \leq s \leq t}\left|p_{i j k_{i}}^{s}-p_{i j}\right|>\frac{\varepsilon}{2 \cdot \mu}\right) \\
& +P\left(\sup _{0 \leq s \leq t}\left|\frac{x_{i k_{i}}(s)}{s}-\mu_{i k_{i}}\right|>\varepsilon\right)
\end{aligned}
$$

$i=1,2, \ldots, J, k_{i}=1,2, \ldots, c_{i}$. 
Finally, we obtain that for $\varepsilon>0$ (see $(3.8)-(3.10))$

$$
\begin{aligned}
\lim _{t \rightarrow \infty} P\left(\frac{\left|W_{j}(t)\right|}{t}>\varepsilon\right) \leq & \sum_{j=1}^{J} \sum_{i=1}^{J} \sum_{k_{i}=1}^{c_{i}} \lim _{t \rightarrow \infty} P\left(\sup _{0 \leq s \leq t}\left|p_{i j k_{i}}^{s}-p_{i j}\right|>\frac{\varepsilon}{c \cdot \mu}\right) \\
& +\sum_{j=1}^{J} \sum_{i=1}^{J} \sum_{k_{i}=1}^{c_{i}} \lim _{t \rightarrow \infty} P\left(\sup _{0 \leq s \leq t}\left|\frac{x_{i k_{i}}(s)}{s}-\mu_{i k_{i}}\right|>\frac{\varepsilon}{c}\right) \\
& +\sum_{j=1}^{J} \sum_{k_{i}=1}^{c_{i}} \lim _{t \rightarrow \infty} P\left(\frac{\sup _{0 \leq s \leq t}\left(x_{i k_{i}}(s)-\tau_{i k_{i}}(s)\right)}{t}>\frac{\varepsilon}{c}\right) \\
\leq & \sum_{j=1}^{J} \sum_{i=1}^{J} \sum_{k_{i}=1}^{c_{i}} P\left(\lim _{t \rightarrow \infty} \sup _{0 \leq s \leq t}\left|p_{i j k_{i}}^{s}-p_{i j}\right|>\varepsilon\right) \\
& +\sum_{j=1}^{J} \sum_{i=1}^{J} \sum_{k_{i}=1}^{c_{i}} P\left(\lim _{t \rightarrow \infty} \sup _{0 \leq s \leq t}\left|\frac{x_{i k_{i}}(s)}{s}-\mu_{i k_{i}}\right|>\varepsilon\right) \\
& +\sum_{j=1}^{J} \sum_{k_{i}=1}^{c_{i}} P\left(\frac{\lim _{t \rightarrow \infty} \sup _{0 \leq s \leq t}\left(x_{i k_{i}}(s)-\tau_{i k_{i}}(s)\right)}{t}>\varepsilon\right) \\
j= & 1,2, \ldots, J .
\end{aligned}
$$

Let us prove that the first term in (3.10) converges to zero. Thus, we get (see the lemma of [34])

$$
\begin{aligned}
P\left(\lim _{t \rightarrow \infty} \sup _{0 \leq s \leq t}\left|p_{i j k_{i}}^{s}-p_{i j}\right|>\varepsilon\right) & \leq P\left(\lim _{t \rightarrow \infty} \lim _{\delta \downarrow 0} \sup _{0 \leq s \leq t}\left|p_{i j k_{i}}^{s}-p_{i j}\right|>\delta\right) \\
& =\lim _{\delta \downarrow 0} P\left(\lim _{t \rightarrow \infty} \sup _{0 \leq s \leq t}\left|p_{i j k_{i}}^{s}-p_{i j}\right|>\delta\right)=0 \\
i, j & =1,2, \ldots, J, k_{i}=1,2, \ldots, c_{i}
\end{aligned}
$$

Using the limit theorem for a renewal process, we see that the second term in (3.10) converges to zero (see [1]). Hence we get that the third term in (3.10) also converges to zero (see Lem. 3.2).

Thus, we prove that (see (3.10) and (3.11))

$$
\sup _{0 \leq s \leq t} \frac{\left|W_{j}(s)\right|}{s} \Rightarrow 0, j=1,2, \ldots, J
$$


Note that (see, for example, [4])

$$
\begin{aligned}
\frac{\hat{x}_{j}(t)-\beta_{j} \cdot t}{t}= & \sum_{i=1}^{J} \sum_{k_{i}=1}^{c_{i}} \frac{\left(x_{i k_{i}}(t)-\mu_{i k_{i}} \cdot t\right) \cdot p_{i j}}{t} \\
& +\frac{\left(A_{j}(t)-\lambda_{j} \cdot t\right)-\left\{\sum_{k_{i}=1}^{c_{i}}\left(x_{i k_{i}}(t)-\mu_{i k_{i}} \cdot t\right)\right\}}{t} \Rightarrow 0, j=1,2, \ldots, J .
\end{aligned}
$$

Thus, using the convergence together theorem (see Thm. 2.1), (3.12) and (3.13), we derive that

$$
\frac{Q_{j}(t)-\beta_{j} \cdot t}{t} \Rightarrow 0, j=1,2, \ldots, J
$$

As a result, we complete the proof of the theorem.

Let us denote $V(t)=\sum_{j=1}^{J} Q_{j}(t), t>0$. Finally, we prove the following limit theorem on the total queue length of jobs in open multiserver queueing networks.

Theorem 3.5. If conditions (2.1)-(2.3) are fulfilled, then

$$
\sup _{0 \leq s \leq t}\left|\frac{V(s)}{s}-\beta\right| \Rightarrow 0 \text {. }
$$

Proof. It suffices to note that $\left|V(t)-\sum_{j=1}^{J} \hat{x}_{j}(t)\right| \leq \sum_{j=1}^{J}\left|W_{j}(t)\right|$ and apply Theorem 3.1. The proof of Theorem 3.2 is complete.

\section{Applications of the main Results}

First of all we present a theorem about the diffusion limit of the queue length of jobs in a multiserver open queueing network.

Theorem 4.1. If conditions (2.1)-(2.3) are satisfied, then

$$
\left(\frac{Q_{1}(n t)-\beta_{1} \cdot n t}{\sqrt{n}} ; \frac{Q_{2}(n t)-\beta_{2} \cdot n t}{\sqrt{n}} ; \ldots ; \frac{Q_{J}(n t)-\beta_{J} \cdot n t}{\sqrt{n}}\right)
$$

$\Rightarrow\left(\hat{\sigma}_{1} \cdot z_{1}(t) ; \hat{\sigma}_{2} \cdot z_{2}(t) ; \ldots ; \hat{\sigma}_{J} \cdot z_{J}(t)\right)$, where $z_{j}(t), \quad j=1,2, \ldots, J, 0 \leq t \leq 1$ are independent standard Wiener processes.

Proof. The proof is based on Lemma 3.1, the convergence together theorem (see Thm. 2.1), and the limit theorem for the renewal process (see, for example, [1]). The proof is now complete.

Next, we present the law of the iterated logarithm for the queue length of jobs in a multiserver open queueing network under heavy traffic conditions. 
Theorem 4.2. If conditions (2.1)-(2.3) are satisfied, then

$$
P\left(\varlimsup_{t \rightarrow \infty} \frac{Q_{j}(t)-\beta_{j} \cdot t}{\hat{\sigma}_{j} \cdot a(t)}=1\right)=P\left(\varliminf_{t \rightarrow \infty} \frac{Q_{j}(t)-\beta_{j} \cdot t}{\widehat{\sigma}_{j} \cdot a(t)}=-1\right)=1,
$$

$j=1,2, \ldots, J$

Proof. This proof is also based on Lemma 3.1, the convergence together theorem (see Thm. 2.1), and the law of the iterated logarithm for the renewal process (see [16]). The proof is now complete.

\section{CONCLUding REMARKS AND FUture RESEARCH}

1. If the conditions of the theorem on SLLN are fulfilled (i.e., conditions (2.1) are satisfied), the network is occupied at first (see Corollary 4.1) and if conditions (2.1) are satisfied later on, the network becomes uncontrollable after a certain time (as $t \geq \max _{1 \leq j \leq J} \max _{1 \leq k_{j} \leq c_{j}} \frac{m_{j k_{j}}}{\beta_{j k_{j}}}$ ) (see Cor. 4.2).

2. Conditions (2.1) are fundamental, - the behaviour of the whole network and its evolution is not clear, if conditions (2.1) are not satisfied. Therefore, this fact is the object of a further research and discussion.

3. The theorems of this paper are proved for a class of multiserver open queueing network in heavy traffic with the service principle "first come, first served", endless waiting time of a customer in each node of the queueing system, and the times between the arrival of customers at the multiserver open queueing networks are independent identically distributed random variables. However, analogous theorems can be applied to a wider class of multiserver open queueing networks in heavy traffic: when the arrival and service of customers in a queue is by groups, when interarrival times of customers at a multiserver open queueing network are weakly dependent random variables, etc.

Acknowledgements. Research supported in part by the National Complex Programme "Theoretical and Engineering aspects of e-service technology creation and application in high-performing calculation platforms"

\section{REFERENCES}

[1] P. Billingsley, Convergence of Probability Measures. Wiley, New York (1968).

[2] A.A. Borovkov, Weak convergence of functionals of random sequences and processes defined on the whole axis. Proc. Stecklov Math. Inst. 128 (1972) 41-65.

[3] A.A. Borovkov, Stochastic Processes in Queueing Theory. Springer, Berlin (1976).

[4] A.A. Borovkov, Asymptotic Methods in Queueing Theory. Wiley, New York (1984).

[5] A.A. Borovkov, Limit theorems for queueing networks. Theory Prob. Appl. 31 (1986) 413427.

[6] H. Chen and A. Mandelbaum, Stochastic discrete flow networks: Diffusion approximations and bottlenecks. The Annals of Probability 19 (1991) 1463-1519. 
[7] C. Flores, Diffusion approximations for computer communications networks. in Computer Communications, Proc. Syrup. Appl. Math., edited by B. Gopinath. American Mathematical Society (1985) 83-124.

[8] P.W. Glynn, Diffusion approximations. in Handbooks in Operations Research and Management Science, edited by D.P. Heyman and M.J. Sobel, Vol. 2 of Stochastic Models. North-Holland (1990).

[9] P.W. Glynn and W. Whitt, A new view of the heavy-traffic limit theorems for infinite-server queues. Adv. Appl. Probab. 23 (1991) 188-209.

[10] B. Grigelionis and R. Mikulevičius, Diffusion approximation in queueing theory. Fundamentals of Teletraffic Theory. Proc. Third Int. Seminar on Teletraffic Theory (1984) 147-158.

[11] J.M. Harrison, The heavy traffic approximation for single server queues in series. Adv. Appl. Probab. 10 (1973) 613-629.

[12] J.M. Harrison, The diffusion approximation for tandem queues in heavy traffic. Adv. Appl. Probab. 10 (1978) 886-905.

[13] J.M. Harrison and A.J. Lemoine, A note on networks of infinite-server queues. J. Appl. Probab. 18 (1981) 561-567.

[14] J.M. Harrison and M.I. Reiman, On the distribution of multidimensional reflected Brownian motion. SIAM J. Appl. Math. 41 (1981) 345-361.

[15] J.M. Harrison and R.J. Williams, Brownian models of open queueing networks with homogeneous customer populations. Stochastics 22 (1987) 77-115.

[16] D.L. Iglehart, Multiple channel queues in heavy traffic. IV. Law of the iterated logarithm. Zeitschrift für Wahrscheinlichkeitstheorie und Verwandte Gebiete 17 (1971) 168-180.

[17] D.L. Iglehart and W. Whitt, Multiple channel queues in heavy traffic I. Adv. Appl. Probab. 2 (1970) 150-175.

[18] D.L. Iglehart and W. Whitt, Multiple channel queues in heavy traffic II: Sequences, networks and batches. Adv. Appl. Probab. 2 (1970) 355-364.

[19] D.P. Johnson, Diffusion Approximations for Optimal Filtering of Jump Processes and for Queueing Networks. Ph.D. dissertation, University of Wisconsin (1983).

[20] F.I. Karpelevitch and A.Ya. Kreinin, Joint distributions in Poissonian tandem queues. Queueing Systems 12 (1992) 274-286.

[21] F.P. Kelly, An asymptotic analysis of blocking. Modelling and Performance Evaluation Methodology. Springer, Berlin (1984) 3-20.

[22] G.P. Klimov, Several solved and unsolved problems of the service by queues in series (in Russian). Izv. AN USSR, Ser. Tech. Kibern. 6 (1970) 88-92.

[23] E.V. Krichagina, R.Sh. Liptzer and A.A. Pukhalsky, The diffusion approximation for queues with input flow, depending on a queue state and general service. Theory Prob. Appl. 33 (1988) 124-135.

[24] Ya.A. Kogan and A.A. Pukhalsky, Tandem queues with finite intermediate waiting room and blocking in heavy traffic. Prob. Control Int. Theory 17 (1988) 3-13.

[25] A.J. Lemoine, Network of queues - A survey of weak convergence results. Management Science 24 (1978) 1175-1193.

[26] R.Sh. Liptzer and A.N. Shiryaev, Theory of Martingales. Kluwer, Boston (1989).

[27] S. Minkevičius, On the global values of the queue length in open queueing networks, Int. J. Comput. Math. (2009) 1029-0265.

[28] S. Minkevičius, On the law of the iterated logarithm in multiserver open queueing networks, Stochastics, 2013 (accepted).

[29] S. Minkevičius and G. Kulvietis, Application of the law of the iterated logarithm in open queueing networks. WSEAS Transactions on Systems 6 (2007) 643-651.

[30] Yu.V. Prohorov, Convergence of random processes and limit theorems in probability theory. Theory Prob. Appl. 1 (1956) 157-214.

[31] M.I. Reiman, Open queueing networks in heavy traffic. Math. Oper. Res. 9 (1984) 441-458.

[32] M.I. Reiman, A multiclass feedback queue in heavy traffic. Adv. Appl. Probab. 20 (1988) 179-207. 
[33] M.I. Reiman and B. Simon, A network of priority queues in heavy traffic: one bottleneck station. Queueing Systems 6 (1990) 33-58.

[34] L. Sakalauskas and S. Minkevičius, On the law of the iterated logarithm in open queueing networks. Eur. J. Oper. Res. 120 (2000) 632-640.

[35] A.V. Skorohod, Studies in the Theory of Random Processes. Addison-Wesley, New York (1965).

[36] V. Strassen, An invariance principle for the law of the iterated logarithm. Zeitschrift für Wahrscheinlichkeitstheorie und Verwandte Gebiete 3 (1964) 211-226.

[37] W. Szczotka and F.P. Kelly, Asymptotic stationarity of queues in series and the heavy traffic approximation. The Annals of Probability 18 (1990) 1232-1248.

[38] W. Whitt, Weak convergence theorems for priority queues: preemptive resume discipline. $J$. Appl. Probab. 8 (1971) 79-94.

[39] W. Whitt, Heavy traffic limit theorems for queues: a survey. in Lecture Notes in Economics and Mathematical Systems, Vol. 98. Springer-Verlag, Berlin, Heidelberg, New York (1971) $307-350$.

[40] W. Whitt, On the heavy-traffic limit theorem for $G I / G / \infty$ queues. Adv. Appl. Probab. 14 (1982) 171-190. 